\title{
Are current staging systems for cancer serving us well?
}

\author{
Peter T Scardino
}

The TNM (tumor, nodes, metastasis) staging system has become the international standard for designating the extent of a cancer and its prognosis. For each organ site and histological type, a cancer is assigned a stage (I, II, III or IV) based on its TNM category and grade. The goals of the system in terms of patient care are to define prognosis, indicate management, and allow comparison of different treatments across institutions. Recently, this system has come under increasing criticism (Roach M et al. [2007] Cancer 109: 213-230).

In prostate cancer, the TNM system fails to include serum PSA levels, the principal tool for screening and early detection as well as the best predictor of pathological stage and prognosis. No physician would treat prostate cancer today without knowing the patient's PSA level. In testicular cancer, the serum markers human chorionic gonadotropin and alpha-feto protein have been incorporated into the TNM system, and the same must be done for PSA in prostate cancer staging if TNM is to remain relevant.

A second valid criticism is the histological classification. The Gleason grade of a prostate cancer is the most accurate way to indicate its degree of differentiation, but is not easily transposed into the classic categories of well, moderate and poor differentiation. The criteria used to define the $\mathrm{T}$ stages and substages are also controversial. Very few cancers are detected incidentally during resection of benign prostatic tissue (cT1a and cT1b). The vast majority detected today are not palpable but are found by needle biopsy for an abnormal PSA. These cT1c cancers vary widely and should be further subdivided by the extent of cancer on biopsy. These imperfections in the TNM staging of prostate cancer can be rectified in the periodical review processes conducted by the International Union Against Cancer (UICC) (Gospodarowicz MK et al. [2004] Cancer 100: 1-5).

Accounting for more serious limitations of the current staging classification schemes would require profound changes. With cancer care ...profound

changes will

have to be

made... iff the

UICC staging

system is

to remain

relevant and

continue to

have....support

of the medical

community.

PT Scardino is

Editor-in-Chief

of Nature Clinical

Practice Urology.

Competing interests

The author declared no

competing interests.

www.nature.com/clinicalpractice doi:10.1038/ncpuro0933 becoming personalized, patients are increasingly interested in their own prognosis, not that of a category. Nomograms, which incorporate continuous variables weighted independently, predict the probability of an event more accurately than stage categories (Steyerberg EW et al. [2007] J Urol 177: 107-112).

The TNM system also fails to recognize cancer as a dynamic, biological process that evolves over time and can be affected by therapy. Scher and Heller recognized this deficiency when they proposed the "Disease States" model, classifying prostate cancers into categories that are managed in similar ways (Scher HI and Heller G [2000] Urology 55: 323-327). "Localized prostate cancers" (NO M0), for example, regardless of $\mathrm{T}$ stage, can be managed with local therapy (surgery, radiation) with the goal of complete eradication. Once the cancer recurs, the patient enters the "rising PSA" state, in which the choice of systemic or local ("salvage") therapy depends as much on the dynamics of PSA (doubling time) as on the original pathological stage and grade of the cancer. If the cancer recurs after androgen deprivation therapy, patients enter the "castrate PSA" state. Finally, they might develop either non-castrate or castrate metastatic cancer. This schema has proven especially useful for designing clinical trials and for guiding management during a phase of the disease when the original TNM stage is no longer relevant.

Neither nomograms nor a disease-states model can be incorporated readily into the current TNM staging system, yet we would have difficulty caring for patients without them. The discovery of specific molecular and genetic changes that define the biology of many cancers will put further pressure on the traditional systems. TNM classification will remain useful for some time, but profound changes will have to be made before long if the UICC staging system is to remain relevant and continue to have the widespread support of the medical community. 